\title{
Weak asymptotic hereditary asphericity for free product and HNN extension of groups
}

\author{
JOANNA JAKUS
}

\begin{abstract}
Asymptotic hereditary asphericity (AHA) is a coarse property of metric spaces and groups, introduced by T Januszkiewicz and J Świątkowski in [3]. Conjecturally, this property is closed under amalgamated free products and HNN extensions over finite subgroups. We prove this conjecture for a slightly weaker property, weak asymptotic hereditary asphericity (AHA $(-))$, which is still strong enough for the purposes which AHA was used for in [3].
\end{abstract}

20F69

\section{Introduction}

Asymptotic hereditary asphericity (AHA) is a property of metric spaces introduced by T Januszkiewicz and J Świa̧tkowski [3]. Roughly, it says that all spheres (of dimension greater than 1) in a Rips complex may be filled by balls in a Rips complex with a larger constant. AHA is a quasiisometry invariant [3, Corollary 3.3], so it is also a well defined property of discrete groups. This property is used in [3] to show the existence of high-dimensional hyperbolic groups which contain no fundamental groups of closed nonpositively curved Riemannian manifolds of dimension greater than two as their subgroups. The examples of such groups are systolic groups, that is groups which act by simplicial automorphisms, properly discontinuously and cocompactly on systolic complexes (some simplicial analogues of CAT(0) spaces). Januszkiewicz and Świątkowski used the fact that every systolic group is AHA (and hence every finitely generated subgroup of a systolic group is AHA), but the fundamental group of a nonpositively curved manifold of dimension greater than two is never AHA.

Apart from the facts mentioned above, not much is known about AHA. It is known that metric spaces which uniformly embed in AHA spaces are also AHA [3, Proposition 3.2]. Another result, [3, Corollary 8.7], tells us that the Cartesian product of more than two infinite groups is never AHA. In [5] the author showed that groups of asymptotic dimension 1 are all AHA. Osajda and Świątkowski [4] prove that the boundary of any AHA group (hyperbolic or CAT(0)) contains no 2-disk. 
There is a natural question, is a free product of two AHA groups also AHA? It would seem that the answer is positive. In fact however, an attempt to prove this conjecture presents severe difficulties. The reason is that we do not have asymptotic analogues of many (even basic) concepts and facts of algebraic topology.

In this paper we introduce weak asymptotic hereditary asphericity $\mathrm{AHA}(-)$, which is a slightly weaker property than AHA. Roughly, instead of filling the spheres in Rips complex by balls, we require that they are filled by some simply connected manifolds. For this new property we prove the following two theorems:

(1) Let $G$ and $H$ be two weakly asymptotically hereditarily aspherical (AHA(-)) groups, and let $K$ be their common finite subgroup. Then $G *_{K} H$ (the free product with amalgamation over $K$ ) also has $\operatorname{AHA}(-)$.

(2) Let $G$ be $\mathrm{AHA}(-)$ group and let $K_{1}$ and $K_{2}$ be isomorphic finite subgroups of $G$. Then the HNN extension of $G, G *\left(K_{1}=K_{2}\right)$, also has $\operatorname{AHA}(-)$.

We also mention that for the purposes set out by Januszkiewicz and Świątkowski [3], for which AHA was invented, AHA(-) is sufficient.

\section{Acknowledgements}

I would like to thank Jacek Świątkowski for suggestions and advice and the referee for useful comments.

The author has previously published under the name Joanna Zubik.

\section{Definition of asymptotic hereditary asphericity}

Recall the definition of asymptotic hereditary asphericity in a form which was introduced by Januszkiewicz and Świątkowski [3]:

Definition 2.1 A metric space $X$ is asymptotically hereditarily aspherical (shortly AHA), if for every $r>0$ there is $R \geqslant r$ such that for every $A \subset X$, any simplicial map $f: S \rightarrow \operatorname{Rips}_{r}(A)\left(S\right.$ is any triangulation of the sphere $S^{k}, k \geqslant 2$ ) has a simplicial extension $F: B \rightarrow \operatorname{Rips}_{R}(A)$ for some triangulation $B$ of the ball $B^{k+1}$ such that $\partial B=S$.

Clearly, the constant $R$ depends only on $r$. We will denote by $R(X, r)$ the value of $R$ in a specific case.

AHA is a quasiisometry invariant property [3, Corollary 3.3]. Thus, it makes sense to speak about AHA for finitely generated groups; we say that a group is AHA if some its Cayley graph is AHA. Below we provide another definition which will turn out to be equivalent for geodesic spaces. 
Definition 2.2 A geodesic space $X$ has the property of AHA* if for every $r>0$ there is $R^{*} \geqslant r$ such that for every path-connected set $A \subset X$, any simplicial map $f: S \rightarrow \operatorname{Rips}_{r}(A)\left(S\right.$ is any triangulation of the sphere $S^{k}, k \geqslant 2$ ) has a simplicial extension $F: B \rightarrow \operatorname{Rips}_{R^{*}}(A)$ for some triangulation $B$ of the ball $B^{k+1}$ such that $\partial B=S$.

In this case the constant $R^{*}$ also depends only on $r$ and we will denote it by $R^{*}(X, r)$.

Lemma 2.3 Let $X$ be a geodesic space. Then $X$ has AHA if and only if it has AHA* .

Proof Clearly AHA implies AHA*. Suppose now $X$ has AHA* We need to check that it has also AHA. Consider any $r, S, A$ and $f: S \rightarrow \operatorname{Rips}_{r}(A)$, as in Definition 2.1. Since $f$ is a continuous function on a connected set, its image is also connected. Therefore $\operatorname{Im}(f)$ is included in one of connected components of $\operatorname{Rips}_{r}(A)$. Let $A_{1} \subset A$ be the set of vertices of this component. Then $\operatorname{Im}(f) \subset \operatorname{Rips}_{r}\left(A_{1}\right)$. Consider the $r$-neighborhood $N_{r}\left(A_{1}\right)=\left\{x \in X:\left(\exists a \in A_{1}\right)(d(a, x) \leqslant r)\right\}$ of $A_{1}$. Such a set is path-connected. To show this, let us notice two things. First, by the definition of $N_{r}\left(A_{1}\right)$, we can connect any point of $N_{r}\left(A_{1}\right)$ with a point of $A_{1}$ by a geodesic which is not longer than $r$ and consequently is included in $N_{r}\left(A_{1}\right)$. Second, notice that any two points $x, y$ from $A_{1}$ can be connected by a geodesic in $N_{r}\left(A_{1}\right)$. Indeed, since Rips ${ }_{r}\left(A_{1}\right)$ is connected, there exists a path along edges which connects the vertices $x$ and $y$. Since $X$ is a geodesic space, for every two adjacent vertices of this path there exists a geodesic which connects them and which is not longer than $r$ (since adjacent vertices in $\operatorname{Rips}_{r}(X)$ are at distance at most $r$ in $X$ ) and consequently is included in $N_{r}\left(A_{1}\right)$. It shows path-connectedness of $N_{r}\left(A_{1}\right)$.

Now, view $f$ as a simplicial map from $S$ to $\operatorname{Rips}_{r}\left(N_{r}\left(A_{1}\right)\right)$. By the assumption that $X$ has AHA $^{*}$, there exists an extension $F^{*}: B \rightarrow \operatorname{Rips}_{R^{*}}\left(N_{r}\left(A_{1}\right)\right)$ of the map $f$, for a constant $R^{*}=R^{*}(X, r)$ and for $B$ such that $\partial B=S$.

For each $x \in N_{r}\left(A_{1}\right)$ choose an element $a_{x}$ from the set $A_{1}$ such that $d\left(x, a_{x}\right) \leqslant r$. For $x \in A_{1}$ we put $a_{x}=x$.

Consider the map $F: V(B) \rightarrow A_{1}$ (with $V(\cdot)$ denoting the vertex set), given by $F(v)=a_{F^{*}(v)}$. Now check the distance between the images of vertices of any edge $[v, w]$ in $B$. We have:

$$
\begin{aligned}
d(F(v), F(w)) & =d\left(a_{F^{*}(v)}, a_{F^{*}(w)}\right) \\
& \leqslant d\left(a_{F^{*}(v)}, F^{*}(v)\right)+d\left(F^{*}(v), F^{*}(w)\right)+d\left(F^{*}(w), a_{F^{*}(w)}\right) \\
& \leqslant r+R^{*}+r=R^{*}+2 r .
\end{aligned}
$$

Hence the map $F$ can be extended to a simplicial map $B \rightarrow \operatorname{Rips}_{R}\left(A_{1}\right) \subset \operatorname{Rips}_{R}(A)$, with $R=R^{*}+2 r$. Therefore $X$ has AHA with the constant $R(X, r)=R$. 


\section{Weak asymptotic hereditary asphericity}

Definition 3.1 A metric space $X$ is weakly asymptotically hereditarily aspherical (AHA(-)) if for every $r>0$ there is $R \geqslant r$ such that for every $A \subset X$, any simplicial map $f: S \rightarrow \operatorname{Rips}_{r}(A)$ ( $S$ is any triangulation of the sphere $S^{k}, k \geqslant 2$ ) has a simplicial extension $F: E \rightarrow \operatorname{Rips}_{R}(A)$ for some triangulation $E$ of a simply connected manifold of dimension $k+1$, such that $\partial E=S$.

AHA(-) is a quasiisometry invariant. A proof of this is analogous to the proof of the fact that AHA is a quasiisometry invariant [3, Corollary 3.3]. As before, for geodesic spaces we have an equivalent definition of $\mathrm{AHA}(-)$ :

Definition 3.2 A geodesic space $X$ has AHA(-) if for every $r>0$ there is $R^{*} \geqslant r$ such that for every path-connected set $A \subset X$, any simplicial map $f: S \rightarrow \operatorname{Rips}_{r}(A)$ ( $S$ is any triangulation of the sphere $S^{k}, k \geqslant 2$ ) has a simplicial extension $F: E \rightarrow$ $\operatorname{Rips}_{R^{*}}(A)$ for some triangulation $E$ of a simply connected manifold of dimension $k+1$, such that $\partial E=S$.

A proof of equivalence of the two above definitions is analogous to the proof of Lemma 2.3. The second definition will be more useful for us. We will use the notation $R^{*}(X, r)$ for the value of $R^{*}$ in a specific case. Clearly such $R^{*}(X, r)$ is not unique.

Remark As we mentioned in Section 1, AHA(-) is sufficient for the purposes [3] for which AHA was invented. Indeed, Januszkiewicz and Świątkowski needed only the fact that systolic groups are $S^{2} \mathrm{FRC}$, which means that for every $r>0$ there is $R \geqslant r$ such that any 2-spherical cycle $f: S \rightarrow \operatorname{Rips}_{r}(X)$ which is nullhomologous in $\operatorname{Rips}_{r}(X)$ has a filling $D$ in $\operatorname{Rips}_{R}(X)$ (ie a 3-chain such that $\partial D$ is the image under $f$ of the fundamental cycle in $S$ ) with the vertex set of $D$ contained in the image under $f$ of the vertex set of $S$; see [3, Section 6]. As we can easily see, $S^{2}$ FRC is implied by AHA as well as by $\mathrm{AHA}(-)$.

\section{One property of maps from spheres to the wedge sum of two spaces}

Let $\left(X, x_{0}\right)$ and $\left(Y, y_{0}\right)$ be based geodesic spaces. Consider the space $X \vee Y$, that is the wedge sum of $X$ and $Y$ (the disjoint union of spaces $X$ and $Y$ after identification 
of $x_{0}$ with $y_{0}$ ). Denote by $p$ the point $x_{0}=y_{0}$ in $X \vee Y$. If $d_{X}$ is the metric on $X$ and $d_{Y}$ is the metric on $Y$, we define a metric $d$ on $X \vee Y$ in the following way:

$$
d(x, y)= \begin{cases}d_{X}(x, y) & \text { if } x, y \in X, \\ d_{Y}(x, y) & \text { if } x, y \in Y, \\ d_{X}\left(x, x_{0}\right)+d_{Y}\left(y_{0}, y\right) & \text { if } x \in X, y \in Y .\end{cases}
$$

Let $S$ be a triangulation of the sphere $S^{k}$ and let $f: S \rightarrow \operatorname{Rips}_{r}(X \vee Y)$ be a simplicial map. Denote by $f^{\prime}$ the map $\left.f\right|_{V(S)}$. We can identify the vertex set of $\operatorname{Rips}_{r}(X \vee Y)$ with the set $X \vee Y$, hence we can treat $f^{\prime}$ as a map from $V(S)$ to $X \vee Y$.

Consider the vertices of the preimage of the set $X \backslash\{p\}$ (analogically, of the set $Y \backslash\{p\}$ ) under $f^{\prime}$. The full subcomplex of $S$ which is spanned on these vertices can consist of several connected components. Such components for $X \backslash\{p\}$ and $Y \backslash\{p\}$ form a family $\mathcal{W}_{f}$ of connected and pairwise disjoint subcomplexes of $S$. The following observation will be crucial for us: there exists a complex $W \in \mathcal{W}_{f}$ that we can enlarge by some vertices from the preimage of $\{p\}$ under $f^{\prime}$ in such a way that its regular neighborhood has connected boundary. In other words, the complement of that neighborhood is connected in $S$.

Before we will show it, we introduce some useful notations and definitions. For a simplicial complex $Z$ we denote by $\operatorname{Bar}(Z)$ its barycentric subdivision. For a subcomplex $Z_{0}$ of a complex $Z$, we denote by $N_{\mathrm{Bar}}\left(Z_{0}, Z\right)$ the subcomplex of $\operatorname{Bar}(Z)$ which is the union of all simplices intersecting $Z_{0}$.

Definition 4.1 A peripheral subcomplex of a simplicial sphere $S$ for a map $f$ is such a subcomplex $W$ of $S$ that

- either $f^{\prime}(V(W)) \subset X$ and $f^{\prime}(v) \in Y$ for each $v$ adjacent to $W$, or $f^{\prime}(V(W)) \subset$ $Y$ and $f^{\prime}(v) \in X$ for each $v$ adjacent to $W$;

- the complement of $N_{\mathrm{Bar}}(W, S)$ in $S$ is connected.

Fact 4.2 There exists a peripheral subcomplex for $f$ in $S$.

Proof Let us consider three cases.

Case 1 There is $W \in \mathcal{W}_{f}$ such that $N_{\mathrm{Bar}}(W, S)$ does not disconnect $S$. Then $W$ is a desired peripheral subcomplex for $f$ in $S$.

Case 2 For every $W \in \mathcal{W}_{f}, N_{\mathrm{Bar}}(W, S)$ disconnects $S$, but there is $W \in \mathcal{W}_{f}$ such that vertices of all subcomplexes from $\mathcal{W}_{f}$ except $W$ lie in one of the connected components of the complement of $N_{\mathrm{Bar}}(W, S)$. It indicates that $f$ maps vertices 
of other components to $p$. Then the full subcomplex spanned on these vertices and vertices of $W$ is peripheral.

Case 3 Previous cases do not occur. Then for every $W \in \mathcal{W}_{f}$, the vertices of subcomplexes from $\mathcal{W}_{f}$ except $W$ lie in at least two connected components of the complement of $N_{\mathrm{Bar}}(W, S)$.

Choose a sequence $W_{n}$ of elements from $\mathcal{W}_{f}$ in the following way. Let $W_{1}, W_{2}$ be any elements from $\mathcal{W}_{f}$. For $i>2$ we choose $W_{i}$ recursively. Assume $W_{i-1}$ is chosen. Vertices of subcomplexes from the family $\mathcal{W}_{f}$ (except for $W_{i-1}$ ) lie in at least two connected components of the complement of $N_{\mathrm{Bar}}\left(W_{i-1}, S\right)$. The subcomplex $W_{i-2}$ is contained in one of them. Choose any element of $\mathcal{W}_{f}$ which is contained in any other component and denote it by $W_{i}$. Notice, that $W_{i}$ is different than $W_{1}, \ldots, W_{i-1}$, because $W_{1}, \ldots, W_{i-3}$ are contained in the same connected component of the complement of $N_{\mathrm{Bar}}\left(W_{i-1}, S\right)$ as $W_{i-2}$.

We obtain an infinite sequence $W_{n}$ of disjoint subcomplexes of $S$, which is impossible since $S$ is the triangulation of a sphere.

Hence, only the first two cases can occur and the proof is complete.

\section{5 АНА(-) for a wedge sum}

Theorem 5.1 If geodesic spaces $X$ and $Y$ have AHA(-), then also $X \vee Y$ has this property. Moreover, for any fixed $r$ the wedge sum $X \vee Y$ satisfies the condition of AHA(-) with the constant $R^{*}(X \vee Y, r)$ equal to the maximum of constants $R^{*}(X, r)$ and $R^{*}(Y, r)$.

Proof Denote by $p$ the basepoint of $X \vee Y$. Fix any $r$ and consider any $A$ and $S$ (a sphere of dimension $k$ ) as in Definition 3.2.

Define $R=\max \left\{R_{x}, R_{y}\right\}$, where $R_{x}:=R^{*}(X, r)$ and $R_{y}:=R^{*}(Y, r)$.

We will consider a map $f: S \rightarrow \operatorname{Rips}_{r}(A)$ together with the map $f^{\prime}\left(\left.f\right|_{V(S)}\right.$ viewed as a map $V(S) \rightarrow A$ ) and the family $\mathcal{W}_{f}$ (of connected disjoint subcomplexes of $S$ ). We will show the existence of an appropriate extension of $f$ by induction on $\left|\mathcal{W}_{f}\right|$.

If $f$ is such that $\left|\mathcal{W}_{f}\right| \leqslant 1$, then $f^{\prime}(V(S)) \subset X$ or $f^{\prime}(V(S)) \subset Y$ and the existence of an appropriate extension of $f$ is an immediate consequence of the fact that $X$ and $Y$ are $\mathrm{AHA}(-)$.

Assume that appropriate extensions exist for maps $f$ such that $\left|\mathcal{W}_{f}\right| \leqslant n$. We will show that we can extend in an appropriate way also $f$ with $\left|\mathcal{W}_{f}\right|=n+1$. If $f^{\prime}(V(S)) \subset X$ 
or $f^{\prime}(V(S)) \subset Y$, then the existence of an appropriate extension of $f$ is again a consequence of the fact that $X$ and $Y$ are $\operatorname{AHA}(-)$. Notice that in the opposite case the basepoint $p$ belongs to $A$. Indeed, if $f^{\prime}(V(S)) \cap X \neq \varnothing$ and $f^{\prime}(V(S)) \cap Y \neq \varnothing$ then let $x \in X$ and $y \in Y$ be points from these intersections respectively. Then $x, y \in A$ and it follows from path-connectedness of $A$ that there exists a path from $x$ to $y$. Every such a path has to pass through the point $p$, so $p \in A$.

Choose a peripheral subcomplex of $S$ for $f$ and denote it by $S_{1}$. Let $S_{2}$ be the subcomplex of $S$ spanned on the rest of vertices. Without loss of generality we can assume that the image of the vertex set of $S_{1}$ by $f^{\prime}$ is included in $X$.

Now consider $\operatorname{Bar}(S)$. Let

$$
S_{0}:=N_{\text {Bar }}\left(S_{1}, S\right) \cap N_{\text {Bar }}\left(S_{2}, S\right) .
$$

$S_{0}$ is equal to $\partial N_{\mathrm{Bar}}\left(S_{i}, S\right)$ (for $i=1,2$ ), so it is a closed manifold of dimension $k-1$. Notice that it may be not a sphere $S^{k-1}$, because $S_{1}$ may be not a disk $D^{k}$.

Define the map $f^{*}: V(\operatorname{Bar}(S)) \rightarrow \operatorname{Rips}_{r}(A)$, such that

$$
f^{*}(v)= \begin{cases}f(v) & \text { for } v \in V(S), \\ p & \text { for } v \in V\left(S_{0}\right), \\ f(w) & \text { for } v \in V(\operatorname{Bar}(S)) \backslash\left(V(S) \cup V\left(S_{0}\right)\right),\end{cases}
$$

where $w$ is any of the vertices from $V(S)$ which are closest to $v$. It is easy to check that the distance in $A$ of the images of vertices of any edge from $\operatorname{Bar}(S)$ is not bigger than $r$. For that reason $f^{*}$ can be extended in a simplicial way to $f^{*}: \operatorname{Bar}(S) \rightarrow \operatorname{Rips}_{r}(A)$.

Let us construct a new complex, starting from $\operatorname{Bar}(S)$. Along $S_{0} \subset \operatorname{Bar}(S)$ we glue $S_{1}^{o}$ (a copy of $N_{\mathrm{Bar}}\left(S_{1}, S\right)$ ), and $S_{2}^{o}$ (a copy of $N_{\mathrm{Bar}}\left(S_{2}, S\right)$ ). Consequently we obtain three spheres: $N_{\mathrm{Bar}}\left(S_{1}, S\right) \cup S_{2}^{o}, S_{2}^{o} \cup S_{1}^{o}, S_{1}^{o} \cup N_{\mathrm{Bar}}\left(S_{2}, S\right)$, whose intersection is $S_{0}$, and the first sphere is glued with the second along $S_{2}^{o}$ and the second sphere is glued with the third along $S_{1}^{o}$.

With the use of $f^{*}$, we will construct auxiliary simplicial maps defined on spheres described above. By the inductive assumption we will be able to extend these maps to simply connected manifolds such that boundaries of these manifolds will be those spheres. With the use of the obtained extensions we will construct an extension of $f^{*}$ which we will use to construct an extension of $f$.

Let $f_{1}^{*}: N_{\mathrm{Bar}}\left(S_{1}, S\right) \cup S_{2}^{o} \rightarrow \operatorname{Rips}_{r}(A)$ be an auxiliary simplicial map defined on vertices as follows:

$$
f_{1}^{*}(v)= \begin{cases}f^{*}(v) & \text { for } v \in V\left(N_{\mathrm{Bar}}\left(S_{1}, S\right)\right), \\ p & \text { for } v \in S_{2}^{o} .\end{cases}
$$


It is clear that $f_{1}^{*}$ is well defined. After identifying $V\left(\operatorname{Rips}_{r}(A)\right)$ with $A$ we observe that $f_{1}^{*}\left[V\left(N_{\mathrm{Bar}}\left(S_{1}, S\right)\right)\right] \subset X \cap A$. Since $f_{1}^{*}\left[V\left(S_{2}^{o}\right)\right]=\{p\}$, the whole image of $\left.f_{1}^{*}\right|_{V\left(N_{\mathrm{Bar}}\left(S_{1}, S\right) \cup S_{2}^{o}\right)}$ is contained in $X \cap A$. Notice that $X \cap A$ is path-connected. Indeed, if $x_{1}, x_{2} \in X \cap A$, then it follows from path-connectedness of $A$ that there exists a path in $A$ from $x_{1}$ to $x_{2}$. If this path is not contained in $X$, then its segments are contained in $Y$. However, every time when such a path exits $X$ or comes back to $X$, it passes also through the point $p$. For that reason every segment, which is contained in $Y$ may be deleted, to obtain a path that is completely contained in $X$.

The space $X$ has AHA(-), so there exists an extension $F_{1}: E_{1} \rightarrow \operatorname{Rips}_{R_{x}}(A \cap X)$ of $f_{1}^{*}$, for some triangulation $E_{1}$ of a simply connected manifold $E_{1}^{k+1}$ such that $\partial E_{1}=N_{\text {Bar }}\left(S_{1}, S\right) \cup S_{2}^{o}$.

Define the next auxiliary map as follows. Let $f_{2}^{*}: N_{\text {Bar }}\left(S_{2}, S\right) \cup S_{1}^{o} \rightarrow \operatorname{Rips}_{r}(A)$ be a simplicial extension of the map defined on the vertex set in the following way:

$$
f_{2}^{*}(v)= \begin{cases}f^{*}(v) & \text { for } v \in V\left(N_{\mathrm{Bar}}\left(S_{2}, S\right)\right) \\ p & \text { for } v \in S_{1}^{o}\end{cases}
$$

It is clear that $f_{2}^{*}$ is well defined. Notice that $\left|\mathcal{W}_{f_{2}^{*}}\right|=n$, so by the inductive assumption there exists an extension $F_{2}: E_{2} \rightarrow \operatorname{Rips}_{R}(A)$ of $f_{2}^{*}$, for some triangulation $E_{2}$ of a simply connected manifold $E_{2}^{k+1}$ such that $\partial E_{2}=N_{\mathrm{Bar}}\left(S_{2}, S\right) \cup S_{1}^{o}$.

Let $B_{o}$ be the simplicial cone over the sphere $S_{1}^{o} \cup S_{2}^{o}$. We will show that $E_{0}:=$ $E_{1} \cup B_{o} \cup E_{2}$ is a simply connected manifold of dimension $k+1$. To do this, we will use Van Kampen's Theorem; see Hatcher [2, Theorem 1.20]. $E_{1}, B_{o}$ and $E_{2}$ are path-connected and simply connected. Also, the intersection of any pair as well as the intersection of all three of these complexes are path-connected. By Van Kampen's Theorem, $\pi_{1}\left(E_{0}\right)$ is trivial, since it is isomorphic to the free product of fundamental groups of complexes $E_{1}, B_{o}$ and $E_{2}$, which are trivial.

Now we will construct an auxiliary map which will be a simplicial extension of $f^{*}$, defined on $E_{0}$. Since $R=\max \left\{R_{x}, R_{y}\right\}$, we have $\operatorname{Rips}_{R_{x}}(A \cap X) \subset \operatorname{Rips}_{R_{x}}(A) \subset$ $\operatorname{Rips}_{R}(A)$, so we can view $F_{1}$ as a function to $\operatorname{Rips}_{R}(A)$. Define $F_{0}: E_{0} \rightarrow \operatorname{Rips}_{R}(A)$ on the vertex set of $E_{0}$ :

$$
F_{0}(v)= \begin{cases}F_{1}(v) & \text { for } v \in V\left(E_{1}\right) \\ F_{2}(v) & \text { for } v \in V\left(E_{2}\right) \\ p & \text { for } v \in V\left(B_{o}\right)\end{cases}
$$

It is easy to see that for edges of $E_{0}$ the distance in $A$ between the images of their vertices is not bigger than $R$. For this reason we can extend $F_{0}$ to a simplicial map. 
In this way we have constructed $E_{0}$-a triangulation of the manifold $E_{0}^{k+1}$ such that the triangulation of the boundary of $E_{0}$ is equal to the barycentric subdivision $\operatorname{Bar}(S)$ of the simplicial sphere $S$ - and we have defined the simplicial map $F_{0}$ on $E_{0}$, which is an extension of $f^{*}$. We will join $E_{0}$ with the product $S^{k} \times[0,1]$ to obtain a manifold with the simplicial sphere $S$ as its boundary.

We triangulate $S^{k} \times\{0\}$ and $S^{k} \times\{1\}$ as $S$; for $v \in V(S)$ let $v^{0}$ (respectively $v^{1}$ ) define the corresponding vertex in $S^{k} \times\{0\}$ (respectively in $S^{k} \times\{1\}$ ). For a simplex $\left[v_{0}, \ldots, v_{i}\right]$ (for $i \leqslant k$ ) $\left[v_{0}^{0}, \ldots, v_{i}^{0}, v_{0}^{1}, \ldots, v_{i}^{1}\right]$ defines a prism in $S^{k} \times[0,1]$. We triangulate each prism in such a way that on $S^{k} \times\{1\}$ we have the initial triangulation, on $S^{k} \times\{0\}$, the barycentric subdivision of $S$, and that we do not have any new additional vertices except for these in $S^{k} \times\{0\}$ (for a precise description of such a triangulation, see Hatcher [2, pages 121-122]). We join $S^{k} \times[0,1]$, triangulated in this way, with $E_{0}$ (by identifying $S^{k} \times\{0\}$ with $\partial E_{0}$ ) and as a result we obtain a manifold with $S$ as a triangulation of its boundary. Denote it by $E$.

Since $\partial E=S, f$ is defined on $\partial E$. We define a simplicial extension $F: E \rightarrow \operatorname{Rips}_{R}(A)$ of $f$, on the vertex set of $E$ :

$$
F(v)= \begin{cases}f(v) & \text { for } v \in V(S) \\ F_{0}(v) & \text { for } v \in V\left(E_{0}\right) .\end{cases}
$$

To see that it is well defined it is sufficient to check what happens for edges $[v, w]$, where $v \in V(S)$ and $w \in V\left(\partial E_{0}\right)$. According to our notation, $v=v^{1}$ and

$$
w \in V\left(\operatorname{Bar}\left[v^{0}, v_{1}^{0}, \ldots, v_{k}^{0}\right]\right)
$$

for some simplex $\left[v, v_{1}, \ldots, v_{k}\right]$ in $S$. Then the distance in $A$ between the images of $v$ and $w$ under $F$ is

$$
\begin{aligned}
d(F(v), F(w))=d\left(f(v), F_{0}(w)\right) & \leqslant \max \left\{d(f(v), f(x)): x \in\left\{v, v_{1}, \ldots, v_{k}\right\}\right\} \\
& \leqslant r,
\end{aligned}
$$

since $f$ is a simplicial map, so $F(v)$ and $F(w)$ span an edge in $\operatorname{Rips}_{R}(A)$.

Consequently, $F$ is a required extension of $f$, which completes the inductive step. This proves that $X \vee Y$ satisfies the condition of AHA(-) with the constant $R^{*}(X \vee Y, r)$ equal to $R=\max \left\{R_{x}, R_{y}\right\}$.

Now consider an infinite iterated wedge sum of spaces $X_{n}(n=1,2, \ldots)$, that is the space

$$
X=\bigcup X_{n}^{\prime}
$$


where $X_{1}^{\prime}:=X_{1}$ and $X_{n+1}^{\prime}:=X_{n}^{\prime} \vee X_{n+1}$ for $n \geqslant 1$ (in this wedge sum we identify any distinguished points of $X_{n}^{\prime}$ and $\left.X_{n+1}\right)$. The metric on $X_{n+1}^{\prime}$, restricted to $X_{n}^{\prime}$, is equal to the metric on $X_{n}^{\prime}$, so we can define a metric on $X$ in the following way: for $x, y \in X$ there exists $n$ such that $x, y \in X_{n}^{\prime}$ and we put $d_{X}(x, y):=d_{X_{n}^{\prime}}(x, y)$.

Theorem 5.2 Let $X$ be an infinite iterated wedge sum of geodesic spaces $X_{n}(n \in \mathbb{N})$,

$$
X=\bigcup X_{n}^{\prime}
$$

Assume that each space $X_{n}$ has AHA(-) and for fixed $r$ the sequence of constants $R^{*}\left(X_{n}, r\right)$ is bounded above by $R_{0}$. Then $X$ has AHA $(-)$. Moreover, for any fixed $r$ the space $X$ satisfies the condition of AHA(-) with the constant $R^{*}(X, r)$ equal to $R_{0}$.

Proof Let us fix any positive real number $r$.

First we observe that for each $n \geqslant 1, X_{n}^{\prime}$ has AHA(-). Indeed, by Theorem 5.1, $X_{2}^{\prime}$ has $\mathrm{AHA}(-)$ and the constant $R^{*}\left(X_{2}^{\prime}, r\right)$ is equal to $\max \left\{R^{*}\left(X_{1}, r\right), R^{*}\left(X_{2}, r\right)\right\} \leqslant R_{0}$. Assume that for $n \leqslant k$ the space $X_{n}^{\prime}$ has AHA(-) and the constant $R^{*}\left(X_{n}^{\prime}, r\right)$ is equal to $R_{0}$. Then, since $X_{k+1}^{\prime}$ is the wedge sum $X_{k}^{\prime} \vee X_{k+1}$, it also has AHA(-) (by Theorem 5.1) and $R^{*}\left(X_{k+1}^{\prime}, r\right)=\max \left\{R_{0}, R_{k+1}\right\}=R_{0}$.

Consider any simplicial map $f: S \rightarrow \operatorname{Rips}_{r}(A)$ for a path-connected set $A \subset X$ and for $S$; a triangulation of $S^{m}, m \geqslant 2$. The triangulation $S$ has always finitely many vertices, so $f[V(S)]$ is contained in $X_{k}^{\prime}$ for some $k$. Notice that the set $A \cap X_{k}^{\prime}$ is path-connected. Indeed, for $x, y \in A \cap X_{k}^{\prime}$ it follows from the path-connectedness of $A$ that there exists a path in $A$ which connects these points. If this path is not contained in $X_{k}^{\prime}$, then notice that every time this path exits $X_{k}^{\prime}$, it has to pass through the basepoint of the wedge sum $X_{k}^{\prime} \vee X_{i}$ for some $i>k$ and afterwards it has to come back also through this point. Consequently, every segment which is not contained in $X_{k}^{\prime}$ can be deleted, to obtain a path which is completely contained in $X_{k}^{\prime}$.

Since $X_{k}^{\prime}$ has AHA(-), there exists $F: E \rightarrow \operatorname{Rips}_{R_{0}}\left(A \cap X_{k}^{\prime}\right) \subset \operatorname{Rips}_{R_{0}}(A)$ for $E$; a triangulation of $E^{m+1}$ such that $\partial E=S$.

\section{$6 \mathrm{AHA}(-)$ for a free product}

Analogously as for AHA, we say that a group is AHA(-) if some its Cayley graph is AHA $(-)$. Since AHA(-) is quasiisometry invariant it is a well defined property for groups. 
Theorem 6.1 Let $G$ and $H$ be groups with AHA(-). Then the free product $G * H$ also has this property.

Proof Fix any generating sets $B_{G}, B_{H}$ for $G, H$ respectively. As a generating set of $G * H$ we take $B_{G} \cup B_{H}$. Consider the Cayley graph of the product $G * H$ with respect to this generating set (we denote it by $\operatorname{Cay}\left(G * H, B_{G} \cup B_{H}\right)$ ).

Notice that $\operatorname{Cay}\left(G * H, B_{G} \cup B_{H}\right)$ is an infinite iterated wedge sum of spaces which are copies of $\operatorname{Cay}\left(G, B_{G}\right)$ and $\operatorname{Cay}\left(H, B_{H}\right)$ (one can see the construction of the Cayley graph of $G * H$ in Cannon [1, page 280], taking trivial $K$ in the amalgamated free product $G *_{K} H$ which is considered there). By Theorem 5.2, Cay $\left(G * H, B_{G} \cup B_{H}\right)$ has $\operatorname{AHA}(-)$, where for fixed $r$ we have

$$
R^{*}\left(\operatorname{Cay}\left(G * H, B_{G} \cup B_{H}\right), r\right)=\max \left\{R^{*}\left(\operatorname{Cay}\left(G, B_{G}\right), r\right), R^{*}\left(\operatorname{Cay}\left(H, B_{H}\right), r\right)\right\} .
$$

We want to prove the analogous theorem for a free product with amalgamation over nontrivial finite subgroup, that is for $G *_{K} H$ with a nontrivial finite $K$.

First, we describe the Cayley graph of $G *_{K} H$. As a generating set for $K$ we take the whole group. Then we extend $K$ to a generating set $B_{G}$ of the group $G$ and to a generating set $B_{H}$ of the group $H$. We take $B_{G} \cup B_{H}$ as a generating set of $G *_{K} H$. Consider the Cayley graph $\operatorname{Cay}\left(G *_{K} H, B_{G} \cup B_{H}\right)$. It is constructed from copies of Cayley graphs of groups $G$ and $H$. We can reconstruct it by taking $\operatorname{Cay}\left(G, B_{G}\right)$ and next gluing along each subgraph corresponding to some left coset of the subgroup $K$ in $G$ the subgraph corresponding to the left coset $e K$ in the graph $\operatorname{Cay}\left(H, B_{H}\right)$ together with the whole new copy of $\operatorname{Cay}\left(H, B_{H}\right)$. In the second step, along each subgraph corresponding to some (other than $e K$ ) left coset of the subgroup $K$ in the glued copy of Cay $\left(H, B_{H}\right)$, we glue the subgraph corresponding to the left coset $e K$ together with a new copy of $\operatorname{Cay}\left(G, B_{G}\right)$. We continue the construction in this fashion. More details of the construction of a Cayley graph of a free amalgamated product can be seen in, eg, Cannon [1, page 280].

Let us modify the graph described above. Since $\operatorname{AHA}(-)$ is a quasiisometry invariant, it suffices to show $\operatorname{AHA}(-)$ for a graph quasiisometric to $\operatorname{Cay}\left(G *_{K} H, B_{G} \cup B_{H}\right)$.

First, modify the graph $\operatorname{Cay}\left(G, B_{G}\right)$. To each subgraph which is contained in this graph and corresponds to some left coset of the subgroup $K$ in $G$ let us add one new vertex and join it by edges with the rest of vertices of the subgraph. Assume that all edges in the graph have length equal to 1 . Denote the resulting graph by $C_{G}$.

Fact 6.2 The graph $C_{G}$ is quasiisometric to the graph $\operatorname{Cay}\left(G, B_{G}\right)$. 
We skip the proof since it is easy to see that the embedding $\operatorname{Cay}\left(G, B_{G}\right) \rightarrow C_{G}$ is a quasiisometry.

We modify the graph $\operatorname{Cay}\left(H, B_{H}\right)$ in the same way as we $\operatorname{did}$ with $\operatorname{Cay}\left(G, B_{G}\right)$, obtaining the graph $C_{H}$.

Now, instead of the graph $\operatorname{Cay}\left(G *_{K} H, B_{G} \cup B_{H}\right)$ consisting of copies of Cay $\left(G, B_{G}\right)$ and copies of Cay $\left(H, B_{H}\right)$ joined in the appropriate way along copies of Cay $(K, K)$, consider a graph which consists of copies of $C_{G}$ and $C_{H}$ joined in single vertices. That is, for each copy of $\operatorname{Cay}\left(G, B_{G}\right)$ and $\operatorname{Cay}\left(H, B_{H}\right)$ in $\operatorname{Cay}\left(G *_{K} H, B_{G} \cup B_{H}\right)$ we consider a copy of $C_{G}$ and $C_{H}$ respectively, and we make the following identifications: If a copy of $\operatorname{Cay}\left(G, B_{G}\right)$ and a copy of $\operatorname{Cay}\left(H, B_{H}\right)$ in $\operatorname{Cay}\left(G *_{K} H, B_{G} \cup B_{H}\right)$ have nonempty intersection (equivalently, their intersection is a copy of $\operatorname{Cay}(K, K)$ ), then their corresponding copies of $C_{G}$ and $C_{H}$ are adjacent in the new graph, meaning that they are joined by identifying appropriate vertices added to copies of $\operatorname{Cay}(K, K)$ in $C_{G}$ and $C_{H}$ (which correspond to a copy of Cay $(K, K)$ mentioned above). Denote this new graph by $C_{G H}$.

Fact 6.3 The graph $C_{G H}$ is quasiisometric to the graph Cay $\left(G *_{K} H, B_{G} \cup B_{H}\right)$.

Proof Since a graph with the standard geodesic metric is quasiisometric to its vertex set (with the restricted metric), it suffices to find a quasiisometry between vertex sets of Cay $\left(G *_{K} H, B_{G} \cup B_{H}\right)$ and $C_{G H}$ (with restricted metrics). Define

$$
f: V\left(\operatorname{Cay}\left(G *_{K} H, B_{G} \cup B_{H}\right)\right) \rightarrow V\left(C_{G H}\right)
$$

in the following way. All copies of $V\left(\operatorname{Cay}\left(G, B_{G}\right)\right)$ and $V\left(\operatorname{Cay}\left(H, B_{H}\right)\right)$, included in $V\left(\operatorname{Cay}\left(G *_{K} H, B_{G} \cup B_{H}\right)\right)$, are mapped by identity to $V\left(C_{G H}\right)$ (onto their corresponding copies). Since each vertex of $V\left(\operatorname{Cay}\left(G *_{K} H, B_{G} \cup B_{H}\right)\right)$ is either a vertex of some copy of $\operatorname{Cay}\left(G, B_{G}\right)$ or of some copy of $\operatorname{Cay}\left(H, B_{H}\right)$, the map $f$ is defined in this way on all vertices.

Let us check that $f$ is a quasiisometric embedding. Take any

$$
x, y \in V\left(\operatorname{Cay}\left(G *_{K} H, B_{G} \cup B_{H}\right)\right) .
$$

It is clear that $d(x, y) \leqslant d(f(x), f(y))$ by the construction of $C_{G H}$ and the definition of $f$. Consider $g(x, y)$, a geodesic with endpoints $x$ and $y$ in $\operatorname{Cay}\left(G *_{K} H, B_{G} \cup B_{H}\right)$. A geodesic $g(f(x), f(y))$ in $C_{G H}$ is not longer than $g(x, y)$ lengthened by two edges in each vertex. The reason is that in all vertices where $g(x, y)$ goes from a copy of $\operatorname{Cay}\left(G, B_{G}\right)$ to a copy of $\operatorname{Cay}\left(H, B_{H}\right)$ (or the opposite) the corresponding path in $C_{G H}$ is two edges longer. These two edges connect a vertex in a copy of $\operatorname{Cay}\left(G, B_{G}\right)$ 
with a vertex in a copy of $\operatorname{Cay}\left(H, B_{H}\right)$ in $C_{G H}$. Thus we have $d(f(x), f(y)) \leqslant$ $|g(x, y)|+2|V(g(x, y))|=3 d(x, y)+2$.

Consequently we have $\frac{1}{3} d(x, y)-2 \leqslant d(x, y) \leqslant d(f(x), f(y)) \leqslant 3 d(x, y)+2$, which shows that $f$ is a quasiisometric embedding.

It remains to check that every vertex in $C_{G H}$ is within a constant distance from the image of $f$. Notice that each vertex of $C_{G H}$ is either in a copy of $V\left(\operatorname{Cay}\left(G, B_{G}\right)\right)$ or $V\left(\operatorname{Cay}\left(H, B_{H}\right)\right.$ ) (so in the image of $f$ ), or it is one of additional vertices (so its distance from the image of $f$ is 1 ). Hence we see that every vertex in $C_{G H}$ is within the distance 1 from the image of $f$. It completes the proof that $f$ is a quasiisometry.

Theorem 6.4 Assume that groups $G$ and $H$ have AHA(-) and that a group $K$ is their common finite subgroup. Then $G *_{K} H$ has also AHA(-).

Proof It suffices to show that the graph $C_{G H}$ has $\mathrm{AHA}(-)$.

Notice that $C_{G H}$ is an infinite iterated wedge sum of spaces which are copies of $C_{G}$ and $C_{H}$. Graphs $C_{G}$ and $C_{H}$ have AHA(-) and they are geodesic spaces (since they are connected graphs). By Theorem 5.2 the space $C_{G H}$ has AHA(-) and for a fixed $r$ the constant $R^{*}\left(C_{G H}, r\right)$ is equal to $\max \left\{R^{*}\left(C_{G}, r\right), R^{*}\left(C_{H}, r\right)\right\}$.

\section{AHA(-) for HNN extension}

To study the behavior of AHA(-) under HNN extensions over finite subgroups let us look at first at a Cayley graph of HNN extension.

Let $K_{1}, K_{2}$ be finite subgroups of a group $G$ and let $\varphi: K_{1} \rightarrow K_{2}$ be an isomorphism. Fix a generating set $B_{G}$ for $G$, which contains $K_{1} \cup K_{2}$. If $G$ has a presentation $\left\langle B_{G} \mid R\right\rangle$, then $\mathrm{HNN}$ extension $G *_{\varphi}$ has the presentation

$$
\left\langle B_{G}, t \mid R, t \varphi(k) t^{-1}=k: k \in K_{1}\right\rangle .
$$

The reader is referred to Cannon [1, page 281] for the detailed description of the graph $\operatorname{Cay}\left(G *_{\varphi}, B_{G} \cup\{t\}\right)$, we only outline the construction. Notice that the graph $\operatorname{Cay}\left(G *_{\varphi}, B_{G} \cup\{t\}\right)$ consists of copies of graphs $\operatorname{Cay}\left(G, B_{G}\right)$ joined in an appropriate way. To reconstruct it take a copy of $\operatorname{Cay}\left(G, B_{G}\right)$. Consider a subgraph of $\operatorname{Cay}\left(G, B_{G}\right)$ corresponding to some left coset of the subgroup $K_{1}$ in $G$. We connect this subgraph with the subgraph corresponding to the left coset $e K_{2}$ in a new copy of $\operatorname{Cay}\left(G, B_{G}\right)$ by a family of edges corresponding to multiplying by $t$, in a manner corresponding to the isomorphism $\varphi$. We do the same for all subgraphs corresponding to left cosets of $K_{1}$ in the initial copy of $\operatorname{Cay}\left(G, B_{G}\right)$. We repeat the analogous process for all 
subgraphs corresponding to the left cosets of $K_{2}$ in the initial copy of $\operatorname{Cay}\left(G, B_{G}\right)$, connecting them with subgraphs corresponding to the left cosets $e K_{1}$ in subsequently added copies of $\operatorname{Cay}\left(G, B_{G}\right)$. We repeat the process for all new copies of $\operatorname{Cay}\left(G, B_{G}\right)$, considering all subgraphs corresponding to left cosets of $K_{1}$ and $K_{2}$ they contain. We continue the construction in this fashion and after infinite number of steps we obtain the graph $\operatorname{Cay}\left(G *_{\varphi}, B_{G} \cup\{t\}\right)$.

Consider the graph with vertices corresponding to copies of Cay $\left(G, B_{G}\right)$ contained in $\operatorname{Cay}\left(G *_{\varphi}, B_{G} \cup\{t\}\right)$ and edges joining the vertices which correspond to copies of $\operatorname{Cay}\left(G, B_{G}\right)$ joined by a family of edges in $\operatorname{Cay}\left(G *_{\varphi}, B_{G} \cup\{t\}\right)$. Such a graph is a tree (see Cannon [1, page 281]).

Instead of the graph $\operatorname{Cay}\left(G *_{\varphi}, B_{G} \cup\{t\}\right)$ we want to consider another graph which is quasiisometric to it. To describe it, consider at first any copy of $\operatorname{Cay}\left(G, B_{G}\right)$ embedded in $\operatorname{Cay}\left(G *_{\varphi}, B_{G} \cup\{t\}\right)$, together with edges which have one endpoint in this copy. As we mentioned, these edges correspond to multiplying by $t$ or $t^{-1}$. We can group them into families in such a way that every family consists of all edges connecting the initial copy of $\operatorname{Cay}\left(G, B_{G}\right)$ with another copy. For each such family separately we take the vertex set, choose all vertices that are not in the initial copy of $\operatorname{Cay}\left(G, B_{G}\right)$ and identify them with each other. Denote the resulting graph by $C_{G}$.

Fact 7.1 The graph $C_{G}$ is quasiisometric to $\operatorname{Cay}\left(G, B_{G}\right)$.

We skip the proof since it is easy to see that the embedding Cay $\left(G, B_{G}\right) \rightarrow C_{G}$ is a quasiisometry.

Now, instead of the graph $\operatorname{Cay}\left(G *_{\varphi}, B_{G} \cup\{t\}\right)$ consisting of copies of $\operatorname{Cay}\left(G, B_{G}\right)$ connected in the appropriate way by families of edges, consider the graph which consists of copies of $C_{G}$ joined in single vertices. That is, for each copy of $\operatorname{Cay}\left(G, B_{G}\right)$ in $\operatorname{Cay}\left(G *_{\varphi}, B_{G} \cup\{t\}\right)$ we consider a copy of $C_{G}$. For two adjacent copies in $\operatorname{Cay}\left(G *_{\varphi}, B_{G} \cup\{t\}\right)$ of $\operatorname{Cay}\left(G, B_{G}\right)$ (meaning these copies are connected by a family of edges) we join their corresponding copies of $C_{G}$ by identifying appropriate vertices (in which edges corresponding to edges from the family mentioned above are glued together). Denote the resulting graph by $C$.

Fact 7.2 The graph $C$ is quasiisometric to $\operatorname{Cay}\left(G *_{\varphi}, B_{G} \cup\{t\}\right)$.

We omit a proof of this fact, because it is a repetition of the proof of Fact 6.3.

Theorem 7.3 Let $K_{1}, K_{2}$ be finite isomorphic subgroups of a group $G$ and let $\varphi$ be an isomorphism between $K_{1}$ and $K_{2}$. If $G$ has $\mathrm{AHA}(-)$, then its HNN extension $G * \varphi$ also has $\mathrm{AHA}(-)$. 
Proof It suffices to show that the graph $C$, which we described above, has AHA(-). Notice at first that $C$ is an infinite iterated wedge sum of spaces which are copies of $C_{G}$. Since $G$ has AHA(-), $C_{G}$ also has this property (as a consequence of Fact 7.1). By Theorem 5.2 the graph $C$ has $\mathrm{AHA}(-)$ and $R^{*}(C, r)=R^{*}\left(C_{G}, r\right)$.

\section{References}

[1] J W Cannon, Geometric group theory, from: "Handbook of geometric topology", (R J Daverman, R B Sher, editors), North-Holland (2002) 261-305 MR1886666

[2] A Hatcher, Algebraic topology, Cambridge Univ. Press (2002) MR1867354

[3] T Januszkiewicz, J Świątkowski, Filling invariants of systolic complexes and groups, Geom. Topol. 11 (2007) 727-758 MR2302501

[4] D Osajda, J Świa̧tkowski, On asymptotically hereditarily aspherical groups arXiv: 1304.7651

[5] J Zubik, Asymptotic hereditary asphericity of metric spaces of asymptotic dimension 1, Topology Appl. 157 (2010) 2815-2818 MR2729340

Instytut Matematyczny, Uniwersytet Wrocławski

pl. Grunwaldzki 2/4, 50-384 Wrocław, Poland

zubik@math.uni.wroc.pl

Received: 7 February 2012 Revised: 23 April 2013 